\title{
Incremental Compression for Image Database
}

\author{
Xi Yin ${ }^{1,2}$ a , Weiqing Huang ${ }^{1, b}$ \\ ${ }^{1}$ Institute of Information Engineering, Chinese Academy of Sciences, Beijing, 100093, China \\ ${ }^{2}$ University of Chinese Academy of Sciences, Beijing, 100040, China \\ aemail: yinxi@iie.ac.cn, bemail: huangweiqing@iie.ac.cn
}

Keywords: incremental image; compression; image database; classify

\begin{abstract}
Image databases obtain the incremental images frequently. However in tradition it is directly compressed to the incremental image, which ignores the redundancy and similarity between images. The general compression way is not satisfied. With an increasing number of compression method proposed, we proposed a method called incremental image compressing method aiming to this question, which is to make the best of the redundancy and similarity between image database and incremental image. Firstly, we tried to classify the incremental image, then seek the most similar image in the class as the reference image, and encode the incremental image using the redundancy and similarity. Simulations test shows the compression results are obviously improved, so the proposed method is superior to traditional compression methods.
\end{abstract}

\section{Introduction}

With the development of science and technology, digital cameras and smart phones have been very popular, people can get a lot of high quality images at any time and any time. Cloud storage and the popularity of the Internet to make the image more convenient. In the face of a large number of image data, storage and transmission has become a difficult problem. As of February 2016, more commonly used in the fields of computer vision, the size of the database are gradually increasing, for example, AWA contains 50 kinds of 30 thousand images [1], ImageNet contains more than 20 thousand kinds of about 14 million images [2], Place2 contains more than and 400 kinds of about 10 million scene images [3]. In the face of the problem of large image database storage, especially the database, the traditional compression coding method can not meet the needs of reality, more effective image compression coding method is imminent.

The traditional intra prediction compression method only uses the redundant information in the incremental image to compress the image. This method only uses the information of spatial redundancy in the image frame, without making full use of the inter frame redundancy, the compression effect can not meet the needs of the data explosion era. In order to use inter frame redundancy compression encoding method based on graph theory to generate pseudo video sequences is proposed in [4-10], but this method in the face image database incremental image, incremental image usually has little or no redundant information between frames, pseudo video sequence compression method in image database incremental image compression effect when compared with the traditional compression encoding method to enhance co.. In order to overcome the pseudo video sequence compression encoding method and traditional compression encoding method, is proposed in this paper, first find the visual and incremental image most similar images from the image database, to find the image as the reference image, the incremental image compression encoding, which can make full use of the redundant information between frames, the compression effect to achieve the optimal. The main contribution of this article is: propose the incremental image oriented image database effective compression encoding method, using classification theory in image database has to find the most similar image as the reference image compression. The remainder of this article is organized as follows. The first part introduces the related work. In the second part, the method of the proposed method is introduced. The third part introduces the experimental setup and analysis of experimental results. The fourth part is the summary of this article. 


\section{Related Work}

The first method to compress the incremental image is HEVC HEVC method (intra coding). This method only uses a single image frame redundancy of single image compression, but not to be able to compress the redundant image frames.

Incremental image compression of the second methods are based on RS (Signal Representative) method, also known as star topology compression. This method first produces RS, the difference between the RS and each incremental image and RS are compressed. The representative methods are MMP[12] Centroid, Method[13] Template and Method[14] MMD[11]. Although these methods also use the redundancy between the images, but there are two shortcomings. First, because of the need to generate RS, the relevance of the incremental image requirements are high, more suitable for remote sensing images and medical images. Second, this kind of star topology structure, RS is fixed as a reference image, when the image compression, the use of redundant information between the frame is not enough.

Incremental image of the third compression methods are pseudo video sequence compression method, also known as the method based on graph theory, more representative of the method is [4-10]. In these methods, firstly, a minimum spanning tree is established, which makes the weights and the minimum of all edges. Among them, the weight of each tree is represented by the parent node as the reference image. Then, the minimum spanning tree is traversed to generate a pseudo video sequence. In the first image of the intra prediction coding, the residual image between the inter frame prediction coding, which is called IPPPP mode. But this method has the following disadvantages in the image database. First, the incremental image, the correlation between the image is very poor, so you can not use the inter frame redundancy. Second, this method does not make full use of the existing image information of the image database.

In this article, an incremental compression coding method for image database is proposed. This method makes full use of the redundant information between the existing image database and incremental image. For each incremental image, the first of its classification, the fastest to find the most similar visual image categories where, through its visual search to find the most similar image in the class, so to find the most similar visual image and incremental image maximum redundancy. Using the most similar image as the reference image, the inter frame prediction coding of the incremental image is performed.

\section{Incremental Compression}

The method proposed in this paper the main purpose is to make full use of the redundancy of existing image database and incremental image, to effectively compress the incremental image (we add to the existing image database is a part of the image is called incremental image). In this section, we describe how to search for the most similar images from the image database through the classification, within class search from the image database. Use visual most similar image as the reference image to compress the incremental image. The advantage of this method is that each incremental image can be found under the condition that the image with the maximum redundancy can be found as the reference image.

Assuming that there is already a large number of images in the local database, the database in accordance with the visual image of the visual similarity of the image classification. Image database to maintain and expand, in the face of such a problem, how to increase the effective compression of the new image. Fortunately, the relationship between the image database and the existing incremental image, make full use of the search to the visual image and the most similar redundancy incremental image, the incremental image as $\mathrm{P}$ frame video encoding of incremental image compression encoding.

Existing image database size may be very large, in order to get the most visually similar images, if you search directly in the image database, will be very expensive. Therefore, this paper proposes a method to classify the incremental image, and then search within the class, which will greatly improve the efficiency of searching the most similar images. A key question is how to correctly 
classify each increment image. Commonly used classification method has SVM (Vector Machine Support), KNN (k-NearestNeighbor) and the depth of learning, etc.. In this method, we first use the existing classification model of the image database to classify the incremental image, which can greatly improve the efficiency of classification. We have not trained a good classification model, we re training classification model for image database. Although the time-consuming re classification model training, but training a classification model, incremental image next to effective, compared each directly from all classes in the image library to search the most similar visual image and high efficiency. For example, the image of the [2] database ImageNe, we can use the existing model AlexNet[15] for incremental image classification, the Place2 database classification can be used in the existing model [16].

After classification, in order to get the most similar images in the class, it should find an effective image distance to measure visual similarity. In this paper, in order to improve the search speed, we directly use the Euclidean distance to measure the similarity of the image. First, feature extraction of image database and incremental image with SPM[17], the distance of all images calculated each incremental image and image database and its corresponding class, and then sort of all distance and incremental image from the nearest image, that is the most similar visual image as the reference image frames.

For an incremental image, this method uses the video coding standard HEVC and the reference image obtained from the 2.2 section to use the incremental image as the inter frame prediction coding for the $\mathrm{P}$ frame. In this way, each image in the incremental image will find the corresponding existing image database in the visual image is the most similar, establish corresponding relation, avoid the incremental image and image database has been separated, considered separately.

\section{Analysis}

In this section, the paper proposes an incremental image compression coding method based on the image database and the method of HEVC intra prediction coding. All experiments based on HEVC platform, QPs (parameters quantization) is set to 22, 27, 32, 37. For HEVC, please refer to [18]. Extracted from 11 videos, according to the visual similarity is divided into 11 categories, each category of images in 23 to 43 , the image is scaled to $256 * 256$. We chose an image from each category as (that is, a total of 11 images) incremental image, the remaining image as an existing image database. In the incremental image, the experiment uses the re training SVM classifier to classify each image. After classification, in the corresponding class by SPM[17] (the dictionary size is 40, the image is divided into 3 layers) for all image feature extraction, using Euclidean distance to calculate the distance of each image and incremental image within class, the nearest image as the reference image, then the incremental image in encoding. HEVC intra prediction coding experiment based on random order of all the incremental image compression coding.

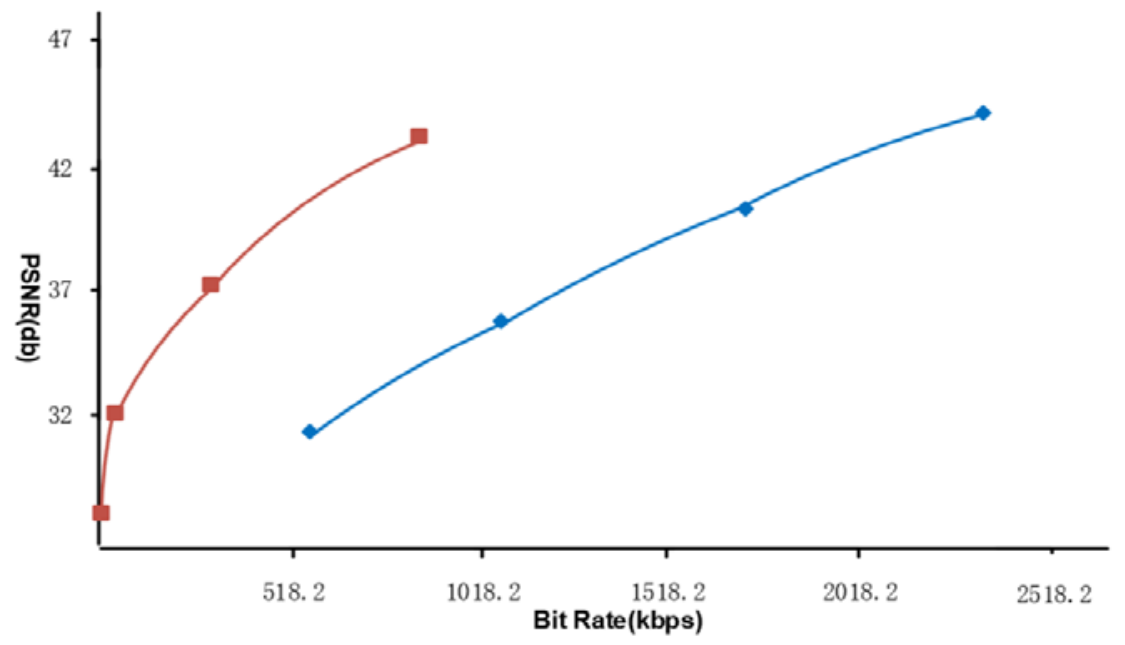

Fig.1. Rate distortion curve of incremental image compression 
The experimental results are shown in figure 1. Figure 1 shows the proposed method and the HEVC intra prediction encoding method for image database incremental image compression encoding rate distortion curve, compared to HEVC intra prediction encoding method, this method significantly improve the compression effect. It shows that the proposed method can make full use of the redundancy of the incremental image and the existing image database, and provide a better reference image for each incremental image.

\section{Conclusion}

In this paper, we propose a novel scheme for incremental compression on the image databases. We attemped to find the most similar image as the reference image in the image databases by classification and intra class searching, then compress the incremental images baesd on the HEVC inter prediction. Our major contribution is improving the compression ratio by making full use of the redundancy between the image databases and the incremental images. Compared with the HEVC intra prediction, the incremental compression coding shows a better compression performance.

\section{References}

[1] Lampert C H, Nickisch H, Harmeling S. Learning to detect unseen object classes by between-class attribute transfer [C]//Computer Vision and Pattern Recognition, 2009. CVPR 2009. IEEE Conference on. IEEE, 2009: 951-958.

[2] J. Deng, A. Berg, S. Satheesh ,et al. ImageNet Large Scale Visual Recognition Competition 2012 (ILSVRC2012). http://www.image-net.org/challenges/LSVRC/2012/

[3] B. Zhou, A. Khosla, A. Lapedriza, et al. Places2: A Large-Scale Database for Scene Understanding [J]. Arxiv, 2015.

[4] Shi Z, Sun X, Wu F. Photo album compression for cloud storage using local features [J]. Emerging and Selected Topics in Circuits and Systems, IEEE Journal on, 2014, 4(1): 17-28.

[5] Chen C P, Chen C S, Chung K L, et al. Image set compression through minimal-cost prediction structures[C] //ICIP. 2004: 1289-1292.

[6] K. Gu, G. Zhai, X. Yang, and W. Zhang. Using free energy principle for blind image quality assessment. IEEE Transactions on Multimedia, 17(1):50-63, 2015.

[7] Shi Z, Sun X, Wu F. Feature-based image set compression[C]//Multimedia and Expo (ICME), 2013 IEEE International Conference on. IEEE, 2013: 1-6.

[8] A. Saha and Q. M. J. Wu. Utilizing image scales towards totally training free blind image quality assessment. IEEE Transactions on Image Processing, 24(6):1879-1892, 2015

[9] Qi X, Tyler J M. A progressive transmission capable diagnostically lossless compression scheme for 3D medical image sets [J]. Information Sciences, 2005, 175(3): 217-243.

[10] Zou R, Au O C, Zhou G, et al. Personal photo album compression and management [C]// Circuits and Systems (ISCAS), 2013 IEEE International Symposium on. IEEE, 2013: 1428-1431.

[11] Karadimitriou K, Tyler J M. The min-max differential method for large-scale storage and compression of medical images[C]//Proceedings of of Annual Molecular Biology and Biotechnology Conference, Baton Rouge, La, USA. 1996.

[12] Karadimitriou K, Tyler J M. Min-max compression methods for medical image databases[J]. ACM SIGMOD Record, 1997, 26(1): 47-52.

[13] Z. Wang and E. P. Simoncelli. Maximum differentiation (MAD) competition: A methodology 
for comparing compu- tational models of perceptual quantities. Journal of Vision, 8(12):8, 2008.

[14] Wang J, Yan H. Form image compression using template extraction and matching[J]. Proceedings of Visual Information Processing, 2000.

[15] Krizhevsky A, Sutskever I, Hinton G E. Imagenet classification with deep convolutional neural networks [C]//Advances in neural information processing systems. 2012: 1097-1105.

[16] Shen L, Lin Z, Huang Q. Learning Deep Convolutional Neural Networks for Places2 Scene Recognition [J]. arXiv preprint arXiv:1512.05830, 2015.

[17] Lazebnik S, Schmid C, Ponce J. Beyond bags of features: Spatial pyramid matching for recognizing natural scene categories [C]//Computer Vision and Pattern Recognition, 2006 IEEE Computer Society Conference on. IEEE, 2006, 2: 2169-2178.

[18] Sullivan G J, Ohm J R, Han W J, et al. Overview of the high efficiency video coding (HEVC) standard[J]. Circuits and Systems for Video Technology, IEEE Transactions on, 2012, 22(12): 1649-1668. 